\title{
Interferencias en la caracterización de residuos cerámicos mediante el ensayo de inhibición de la luminiscencia
}

\author{
M.F. GAZULLA, M.P. GÓMEZ, E. MONFORT, M. ORDUÑ̃ \\ Instituto de Tecnología Cerámica. Asociación de Investigación de las Industrias Cerámicas. Universitat Jaume I. Castellón. España.
}

\begin{abstract}
En este trabajo se estudia la influencia de diferentes factores sobre los resultados obtenidos en la determinación de la toxicidad de muestras de residuos mediante el ensayo de inhibición de la luminiscencia de la bacteria Photobacterium phosphoreum (Vibrio fischeri), llegándose a la conclusión de que este método, por sí sólo, no es adecuado para caracterizar residuos de la industria cerámica.
\end{abstract}

Palabras clave: Caracterización de residuos, ensayo de luminiscencia, industria cerámica.

Interferences in ceramic waste characterisation by the luminescence inhibition assay

The present study was undertaken to determine the influence of different factors on the results obtained in the determination of the toxicity of ceramic wastes by Photobacterium phosphoreum (Vibrio fischeri) bacteria luminescence inhibition assay. The study indicates that this method, used just by itself, is inadequate for characterising ceramic industry waste.

Keywords: Waste characterisation, luminescence assay, ceramic industry.

\section{INTRODUCCIÓN}

La toxicidad es un parámetro primordial en la evaluación del impacto ambiental de una corriente contaminante, ya que las determinaciones físicas y químicas solas pueden resultar insuficientes para la evaluación de los potenciales efectos sobre los seres vivos. La forma de obtener una mayor información del impacto de un determinado residuo sobre el medio ambiente es registrar el efecto producido sobre un ser vivo. Existe una gran variedad de bioensayos para evaluar la toxicidad de una sustancia utilizando diferentes organismos (algas, fitoplácton, protozoos, microcrustáceos, insectos, peces, conejos, etc.).

En la legislación española en el apéndice IV de la Orden Ministerial del 13 de octubre de 1989 se recogen los métodos de caracterización de residuos tóxicos y peligrosos (código $\mathrm{H})$ [1]. Entre las condiciones que establece esta normativa para que un residuo sea peligroso se encuentra evaluar la toxicidad de los lixiviados del residuo. En esta disposición se admite la utilización indistinta de dos bioensayos: el bioensayo de luminiscencia de la bacteria Photobacterium phosphoreum (Vibrio fischeri) o el bioensayo de inhibición de la movilidad de la daphnia magna. En la práctica el primero ha tenido una mayor implantación debido a la sencillez en su ejecución e interpretación.

La determinación de la toxicidad mediante este bioensayo se basa en medir el grado de reducción de luz que se produce al poner en contacto diferentes concentraciones de una muestra problema con bacterias Vibrio fischeri, asociándose dicha disminución de luz a una inhibición del proceso metabólico de las bacterias, y por tanto, a la toxicidad de la muestra [2-5]. El resultado del bioensayo se expresa como la concentración de muestra que reduce la intensidad inicial de luz en un
$50 \%$ tras un tiempo de contacto de 15 minutos a $15^{\circ} \mathrm{C}$ (valor denominado $\mathrm{EC}_{50}$ ).

Los estudios de toxicidad generalmente se llevan a cabo con compuestos químicos de forma individual y bajo condiciones estandarizadas. Una misma sustancia puede dar valores diferentes de $\mathrm{EC}_{50}$ si existen interacciones sinérgicas o antagónicas con los constituyentes del medio de análisis [6-8].

En algunos estudios realizados por el ITC [9-12] con residuos de la industria cerámica se ha constatado que no existe una relación sencilla entre la toxicidad determinada mediante el ensayo de bioluminiscencia y la presencia de elementos tóxicos en los lixiviados, por lo que se ha considerado interesante realizar un estudio de los principales factores que influyen sobre la determinación de los valores obtenidos para la toxicidad en este ensayo.

Los residuos de la industria cerámica potencialmente más tóxicos son los generados en las etapas de preparación y aplicación de esmaltes, que están constituidos fundamentalmente por restos de esmaltes. Los principales elementos presentes en los lixiviados obtenidos a partir de estos residuos en la industria de baldosas cerámicas esmaltadas son el plomo, el cinc y el boro [10].

\section{OBJETO}

El objeto de este trabajo es estudiar los factores que pueden afectar al valor de la $\mathrm{EC}_{50}$ obtenido mediante el ensayo de inhibición de la luminiscencia de la bacteria Photobacterium phosphoreum (Vibrio fischeri), aplicada a la caracterización de muestras de residuos que contengan esmaltes cerámicos. 


\section{DESARROLLO EXPERIMENTAL}

\subsection{Determinación de la $\mathrm{EC}_{50}$}

El ensayo de inhibición de la luminiscencia se ha realizado midiendo la emisión de luz de una suspensión de bacterias, antes y después de un tiempo de contacto de 15 minutos a una temperatura de $15^{\circ} \mathrm{C}$ con una serie de soluciones preparadas a partir de una inicial diluida con $\mathrm{NaCl}$ al $2 \%$.

Cada una de estas diluciones de la muestra de ensayo provoca un porcentaje de inhibición de la luz, ya que existe una relación concentración-efecto, de esta forma, mediante un análisis de regresión lineal se calcula aquella concentración que ha provocado un $50 \%$ de inhibición de luz del microorganismo, que es el valor de $\mathrm{EC}_{50}$.

$\mathrm{La}^{\mathrm{EC}_{50}}$ normalmente se expresa como la concentración de disolución o de lixiviado (en la caracterización de residuos) a partir de la cual se toman fracciones para obtener una inhibición del 50\% de la luminiscencia. No obstante en este trabajo cuando se estudia el efecto de los metales, la $\mathrm{EC}_{50}$ se ha expresado como la concentración del elemento en la disolución en contacto con las bacterias que produce una inhibición de la luminiscencia del 50\%. Esta forma de expresión permite comparar con facilidad los resultados de este trabajo, aunque se haya partido de disoluciones con diferentes concentraciones iniciales del elemento estudiado.

\subsection{Materiales y equipos}

Las bacterias utilizadas para la realización del ensayo de bioluminiscencia han sido bacterias del tipo Vibrio Fischeri, que se suministran deshidratadas y congeladas y con una solución que contiene los nutrientes necesarios para reactivar la suspensión bacteriana. Los tests de bacterias luminiscentes utilizados para la realización de este trabajo fueron: LCK-480 Vibrio Fischeri NRLL-B-11177 No lote: 110711 y 06132, y han sido suministrados por la firma NEURTEK MEDIO AMBIENTE.

La solución con la que se diluye la suspensión bacteriana es una solución de $\mathrm{NaCl}$ al $2 \%$ en agua destilada. Los materiales de referencia empleados para la validación del método han sido: solución de $25 \mathrm{mg} \mathrm{L}^{-1}$ de $\mathrm{Zn}^{+2}$ preparada a partir de $\mathrm{ZnSO}_{4} \cdot 7 \mathrm{H}_{2} \mathrm{O}$, solución de $6 \mathrm{mg} \mathrm{L}^{-1}$ de 3,5-diclorofenol $\left(\mathrm{C}_{6} \mathrm{H}_{4} \mathrm{OCl}_{2}\right)$ y solución de $\mathrm{mg} \mathrm{L}^{-1}$ de $\mathrm{Cr}^{+6}$ preparada a partir de $\mathrm{K}_{2} \mathrm{Cr}_{2} \mathrm{O}_{7}$.

Las soluciones de calcio, boro, cinc y plomo, objeto de estudio, se prepararon a partir de producto Merck R.A.

$\mathrm{El} \mathrm{pH}$ se determinó con un pHmetro/ionómetro modelo 692 de la firma METROHM.

La medida de la luz emitida por las bacterias se realizó con un luminómetro GEM BIOMEDICAL modelo OPTOCOMP I provisto de un incubador capaz de mantener la temperatura a $15 \pm 1^{\circ} \mathrm{C}$.

\subsection{Variables estudiadas}

Aunque el plomo es un elemento que tiende a ser eliminado en los esmaltes de la industria cerámica por su toxicidad, en este trabajo se ha tomado como base para estudiar el efecto de diferentes variables sobre los resultados obtenidos al realizar una caracterización de un residuo cerámico, basándose en el ensayo de luminiscencia con la bacteria Vibrio Fischeri.

La concentración de plomo de la solución objeto de estudio, a la cual se irán modificando variables, ha sido de $10 \mathrm{mg}$ $\mathrm{L}^{-1}$. La concentración de plomo en la solución se ha establecido de forma que se produjese una inhibición de la luminiscencia en el intervalo de trabajo estudiado.

Las variables que se han estudiado son:

i) $\mathrm{pH}$ del medio,

ii) presencia de materia orgánica,

iii) presencia de otros cationes como $\mathrm{Zn}$, B y Ca en una solución con una concentración fija de plomo.

\subsubsection{EFECTO DEL pH DEL MEDIO}

Para el estudio de la influencia del $\mathrm{pH}$ se prepararon dos series de soluciones: una serie simplemente constaba de soluciones de $\mathrm{NaCl}$ al $2 \%$, en las que únicamente cambiaba el valor del $\mathrm{pH}$, y en la otra serie las soluciones contenían $10 \mathrm{mg}$. $\mathrm{L}^{-1}$ de plomo en medio $\mathrm{NaCl}$ al $2 \%$. El ajuste del $\mathrm{pH}$ de las soluciones de ensayo se llevó a cabo con $\mathrm{HCl}$ al $10 \%$ y / o NaOH al $10 \%$.

TABLA 1. SOLUCIONES PREPARADAS PARA EL ESTUDIO DEL EFECTO DEL PH.

\begin{tabular}{|c|c|c|c|c|c|}
\hline & \multicolumn{5}{|c|}{ Valor de $\mathbf{p H}$ de las disoluciones } \\
\hline Solución $\mathrm{NaCl}$ 2\% & 2 & 3 & 5 & 7 & 11 \\
\hline $\begin{array}{c}\text { Solución 10 mg.L } \\
\text { en } \mathbf{~ N a C l} \text { 2\% }\end{array}$ & 2 & 3 & 5 & 7 & 11 \\
\hline
\end{tabular}

Las especies de plomo predominantes en la disolución a cada valor de $\mathrm{pH}$ se encuentran descritas en la bibliografía $[13,14]$.

\subsubsection{EFECTO DE LA MATERIA ORGÁNICA}

En este caso se prepararon dos series de soluciones con diferentes concentraciones de materia orgánica (expresada como $\mathrm{mg} \mathrm{O}_{2} \cdot \mathbf{L}^{-1}$ ), que se adicionó en forma de glucosa y ácido glutámico. Se eligió un intervalo de concentraciones de materia orgánica representativo del que se encuentra en los residuos procedentes de la industria cerámica.

Para realizar el estudio se preparó una primera serie de soluciones que contenía la matriz de $\mathrm{NaCl}$ al $2 \%$, y otra serie que contenía además $10 \mathrm{mg} \cdot \mathrm{L}^{-1}$ de plomo (Tabla 1 ).

TABLA 2. SOLUCIONES PREPARADAS PARA EL ESTUDIO DEL EFECTO DE LA MATERIA ORGÁNICA.

\begin{tabular}{|c|c|c|c|c|c|c|}
\hline & \multicolumn{6}{|c|}{$\begin{array}{l}\text { Concentración de materia orgánica [mg } \\
\left.\qquad \mathrm{O}_{2} \cdot \mathrm{L}^{-1}\right]\end{array}$} \\
\hline Solución $\mathrm{NaCl} 2 \%$ & 0 & 63 & 125 & 250 & 1000 & 5000 \\
\hline $\begin{array}{c}\text { Solución } 10 \mathrm{mg}^{-\mathrm{L}^{-1}} \text { de } \mathrm{Pb} \\
\text { en } \mathrm{NaCl} 2 \%\end{array}$ & 0 & 63 & 125 & 250 & 1000 & 5000 \\
\hline
\end{tabular}

\subsubsection{EFECTO DE LA PRESENCIA DE CATIONES EN EL MEDIO}

Aparte de los elementos presentes en los lixiviados de la industria cerámica comentados anteriormente - plomo, cinc y boro-, se ha incluido el estudio del calcio para considerar el 
posible efecto que puede causar un cambio importante en la dureza del agua o en la solubilidad de las fritas [10].

Para realizar el estudio se prepararon diferentes soluciones en medio $\mathrm{NaCl}$ al $2 \%$ y el valor del $\mathrm{pH}$ se ajustó a 7 . En estas soluciones se introdujeron los cationes objeto de estudio de forma individual y mezclados con otros, las combinaciones ensayadas se ofrecen en la Tabla 3.

TABLA 3. SOLUCIONES ENSAYADAS PARA EL ESTUDIO DEL EFECTO DEL PLOMO, CINC, BORO Y CALCIO SOBRE LA TOXICIDAD DE UNA DISOLUCIÓN DE PLOMO.

\begin{tabular}{|c|c|c|c|c|}
\hline \multicolumn{5}{|c|}{ Composición de las soluciones empleadas (NaCl al 2\% y pH=7) } \\
\hline \multirow{2}{*}{ Composición } & \multicolumn{4}{|c|}{ Concentración de metales (en mg. $\mathrm{L}^{-1}$ ) } \\
\cline { 2 - 5 } & $\mathrm{Pb}$ & $\mathrm{Zn}$ & $\mathbf{B}$ & $\mathrm{Ca}$ \\
\hline $\mathbf{1}$ & 10 & & & \\
\hline $\mathbf{2}$ & & 10 & & \\
\hline $\mathbf{3}$ & & & 1000 & \\
\hline $\mathbf{4}$ & 10 & 10 & & \\
\hline $\mathbf{5}$ & 10 & 10 & 10 & \\
\hline $\mathbf{6}$ & 50 & & & 100 \\
\hline $\mathbf{7}$ & 50 & & & 500 \\
\hline
\end{tabular}

En todos los ensayos la concentración del elemento estudiado se ha ajustado de forma que se produjese una inhibición de la luminiscencia. En este sentido para las mezclas 3, 6 y 7 se han aumentado las concentraciones de boro y plomo de forma que permitiesen el cálculo de la $\mathrm{EC}_{50}$ sin realizar extrapolaciones.

\section{RESULTADOS Y DISCUSIÓN.}

\subsection{Valor de $\mathrm{EC}_{50}$ del plomo, cinc y boro.}

Para comparar los resultados obtenidos se ensayó cada catión en soluciones individuales (composiciones 1, 2 y 3 de la tabla 3) en medio $\mathrm{NaCl}$ al $2 \%$ y pH 7. Los resultados obtenidos se recogen en la Tabla 4.

TABLA 4. VALORES DE EC $_{50}$ OBTENIDOS PARA LAS SOLUCIONES MONOELEMENTALES DE PLOMO, CINC Y BORO

\begin{tabular}{|c|c|c|}
\hline Composición & Elemento estudiado & $\mathrm{EC}_{50}\left(\mathrm{mg}\right.$ elemento $\left.\cdot \mathrm{L}^{-1}\right)$ \\
\hline 1 & $\mathrm{~Pb}$ & $5.5 \pm 0.6$ \\
\hline 2 & $\mathrm{Zn}$ & $1.4 \pm 0.3$ \\
\hline 3 & $\mathrm{~B}$ & $87 \pm 7$ \\
\hline
\end{tabular}

Se observa una diferencia notable entre los valores de $\mathrm{EC}_{50}$ para los tres elementos estudiados. El cinc es el catión con una $\mathrm{EC}_{50}$ más baja lo que significa que es el más tóxico de los tres cationes para las bacterias, ya que produce una inhibición de la luminiscencia con una menor concentración, resultando el boro el elemento menos tóxico según este ensayo.

\subsection{Efecto del pH del medio}

Previamente al ensayo de la determinación de la $\mathrm{EC}_{50}$ de la solución de plomo se realizaron ensayos de disoluciones de $\mathrm{NaCl}$ al $2 \%$, en los que se observó que en las disoluciones con $\mathrm{pH}$ inferior a 5 se produce una inhibición de la luminiscencia, y a pH del orden de 7 o superiores no se produce inhibición. Estos resultados están de acuerdo con los encontrados en la bibliografía [8], y demuestran una clara influencia del $\mathrm{pH}$ del medio en el ensayo de determinación de la toxicidad, independientemente del resto de constituyentes de la muestra.

TABla 5: VALORES DE EC $_{50}$ OBTENIDOS PARA UNA SOLUCiÓN DE 10 MG L-1 DE PB EN NACl $2 \%$ EN FUNCIÓN DEL PH

\begin{tabular}{|c|c|}
\hline $\mathbf{p H}$ & $\mathbf{E C}_{50}\left(\mathbf{m g ~ P b} \cdot \mathbf{L}^{-1}\right)$ \\
\hline 2 & $<0.1$ \\
\hline 3 & $<0.2$ \\
\hline 5 & $5.2 \pm 0.5$ \\
\hline 7 & $5.4 \pm 0.6$ \\
\hline 11 & No inhibe \\
\hline
\end{tabular}

En los ensayos realizados para estudiar el efecto del $\mathrm{pH}$ sobre una disolución que contiene plomo, cuyos resultados se detallan en la tabla 5, se observa que entre pH 5 y 7 no existe una diferencia significativa entre los valores de EC50. Sin embargo, cuando el $\mathrm{pH}$ de la disolución es 11, no se produce inhibición de la luminiscencia, y cuando el $\mathrm{pH}$ es inferior a 3 la toxicidad de la disolución aumenta de forma considerable, aunque a este $\mathrm{pH}$, una solución de $\mathrm{NaCl}$ al $2 \%$ es tóxica, tanto en presencia como en ausencia de plomo. Esto puede estar relacionado con las especies de plomo presentes en la solución a cada valor de $\mathrm{pH}$. Teniendo en cuenta los diagramas de estabilidad del plomo en función del $\mathrm{pH}[13,14]$, cuando el $\mathrm{pH}$ es inferior a 8, la especie predominante en solución es $\mathrm{Pb}^{2+}$, mientras que a pH 11 la especie predominante en solución es $\mathrm{Pb}(\mathrm{OH})_{2}$ precipitado.

Desde el punto de vista de la caracterización de residuos esto significa que al ajustar el $\mathrm{pH}$ antes de medir la toxicidad, tal y como establece la norma de ensayo [2], se pueden modificar las características de la muestra inicial dando lugar a resultados muy diferentes a los que se obtendrían con la muestra problema inicial. En la caracterización de residuos de la industria cerámica los valores típicos del $\mathrm{pH}$ en los lixiviados obtenidos según el procedimiento de lixiviación EP (método 1 de la legislación española), suelen ser del orden de 5 a 7 [9-10], intervalo en el que, según los resultados obtenidos, la variación de la EC50 con el pH para una disolución de plomo es poco significativa.

\subsection{Efecto de la materia orgánica}

Siguiendo la metodología aplicada en el apartado anterior se realizaron ensayos en disoluciones que únicamente contenían materia orgánica en medio $\mathrm{NaCl}$ al 2\% (0-5000 $\left.\mathrm{mg} \mathrm{O}_{2} \cdot \mathrm{L}^{-1}\right)$, en los que se observó que en ningún caso se producía una inhibición de la luminiscencia.

En la tabla 6 se muestran los resultados obtenidos en los ensayos realizados para el estudio del efecto de la materia orgánica adicionada (glucosa y ácido glutámico) sobre la toxicidad de una disolución que contiene plomo, apreciándose que existe una variación importante del valor de $\mathrm{EC}_{50}$ del plomo en función de la concentración de materia orgánica. En efecto, para valores bajos de materia orgánica $\left(63 \mathrm{mg} \mathrm{O} \cdot \mathrm{L}^{-1}\right)$ el valor obtenido es el mismo que para una disolución sin materia orgánica, pero a concentraciones superiores a $125 \mathrm{mg} \mathrm{O}_{2} \cdot \mathrm{L}^{-1}$ se observa un aumento considerable del valor del $\mathrm{EC}_{50^{\prime}} \mathrm{o}$ lo que es lo mismo, una disminución de la toxicidad. 
TABLA 6: VALORES DE EC50 OBTENIDOS PARA UNA SOLUCIÓN DE $10 \mathrm{mg} \cdot \mathrm{L}^{-1}$ DE Pb EN NaCl 2\% EN FUNCIÓN DE LA MATERIA ORGÁNICA.

\begin{tabular}{|c|c|}
\hline Materia orgánica $\left(\mathbf{m g O}_{2} \cdot \mathbf{L}^{-1}\right)$ & $\mathbf{E C}_{50}\left(\mathbf{m g ~ P b} \cdot \mathbf{L}^{-1}\right)$ \\
\hline 63 & $5.4 \pm 0.5$ \\
\hline 125 & $8.2 \pm 0.8$ \\
\hline 250 & $18 \pm 2$ \\
\hline 1000 & $12.5 \pm 1.1$ \\
\hline 5000 & $9.0 \pm 1.0$ \\
\hline
\end{tabular}

En este caso se ha observado que la materia orgánica adicionada hasta $250 \mathrm{mgO} \cdot \mathrm{L}^{-1}$ ha actuado como un potenciador de la luminiscencia, probablemente por ser un nutriente para las bacterias; obviamente para otro tipo de materia orgánica puede suceder lo contrario. Pero, en cualquier caso el aspecto más importante es que en la caracterización de residuos generados en la industria cerámica de productos esmaltados, el contenido y la naturaleza de la materia orgánica presente en los fangos obtenidos en la fase de preparación y aplicación de esmaltes es muy variable, y puede modificar sustancialmente los resultados de caracterización de residuos obtenidos en este ensayo para una misma carga contaminante, lo que puede conllevar que se obtengan resultados contradictorios.

\subsection{Efecto de la presencia de cationes en el medio}

En la tabla 7 se muestran los resultados obtenidos en los ensayos realizados para el estudio del efecto de diferentes metales sobre la determinación de la $\mathrm{EC}_{50}$. La $\mathrm{EC}_{50}$ se ha expresado en todos los casos como la concentración del cation plomo que produce una inhibición del $50 \%$ de la luminiscencia, partiendo de diferentes soluciones.

TABLA 7: VALORES DE EC $_{50}$ EN DIFERENTES MATRICES.

\begin{tabular}{|c|c|c|}
\hline Composición & Soluciones de partida en $\mathbf{N a C l} \mathbf{2} \%$ & $\begin{array}{c}\mathrm{EC}_{\mathbf{5 0}} \mathbf{~} \mathbf{m g} \\
\left.\mathbf{P b} \cdot \mathbf{L}^{-1}\right)\end{array}$ \\
\hline $\mathbf{4}$ & $10 \mathrm{mg} . \mathrm{L}^{-1} \mathrm{~Pb}+10 \mathrm{mg} \cdot \mathrm{L}^{-1} \mathrm{Zn}$ & $1.8 \pm 0.3$ \\
\hline $\mathbf{5}$ & $10 \mathrm{mg} \cdot \mathrm{L}^{-1} \mathrm{~Pb}+10 \mathrm{mg} \cdot \mathrm{L}^{-1} \mathrm{Zn}+10 \mathrm{mg} \cdot \mathrm{L}^{-1} \mathrm{~B}$ & $1.9 \pm 0.3$ \\
\hline $\mathbf{6}$ & $50 \mathrm{mg} \cdot \mathrm{L}^{-1} \mathrm{~Pb}+100 \mathrm{mg} \cdot \mathrm{L}^{-1} \mathrm{Ca}$ & $15.1 \pm 1.1$ \\
\hline $\mathbf{7}$ & $50 \mathrm{mg} \cdot \mathrm{L}^{-1} \mathrm{~Pb}+500 \mathrm{mg} \cdot \mathrm{L}^{-1} \mathrm{Ca}$ & $10.6 \pm 0.9$ \\
\hline
\end{tabular}

De los resultados que se presentan en las tablas 4 y 7 se puede establecer los siguientes comentarios:

- El efecto de los cationes sobre el $\mathrm{EC}_{50}$ no es aditivo y la toxicidad de la muestra depende casi exclusivamente de la concentración del elemento tóxico (comparar resultados de la composición 2 de la tabla 4 con las composiciones 4 y 5 de la tabla 7).

- Al estudiar el efecto de la presencia de calcio sobre el valor de la $\mathrm{EC}_{50}$ (composiciones 6 y 7) se observa que este elemento produce una disminución importante de la toxicidad de la disolución que sólo contiene plomo (composición 1).

4.5 Valoración de los resultados al aplicar este ensayo para la caracterización de residuos según la legislación española.

Para evaluar la toxicidad de los residuos según este ensayo, en la legislación española se establece que para que un residuo sea tóxico el lixiviado obtenido de dicho residuo debe presentar un valor de $\mathrm{EC}_{50} \leq 3000 \mathrm{mg} \mathrm{L}^{-1}$, es decir, un residuo será tóxico si la adición de una cantidad inferior al $0.3 \%$ de lixiviado produce una inhibición del $50 \%$ de la bioluminiscencia.

Para poner de manifiesto la importancia de los resultados obtenidos se considera interesante poner un ejemplo. A partir del residuo $\mathrm{R}$ de la industria cerámica se ha obtenido un lixiviado que contiene fundamentalmente como contaminante plomo, en una concentración del orden de $3000 \mathrm{mg} \mathrm{L}^{-1}$. La adición de un $0.3 \%$ de este lixiviado dará lugar a una concentración de $9 \mathrm{mg} \mathrm{L}^{-1}$ de plomo en la solución en contacto con las bacterias, por tanto dado que este valor es superior a $5.3 \mathrm{mg} \mathrm{L}^{-1}$ el residuo R será tóxico según la legislación española.

Sin embargo, el residuo R podría llegar a ser considerado no tóxico si en el medio existiese materia orgánica del tipo y cantidades como algunas de las ensayadas en el apartado 4.3, o si en el lixiviado existiesen cantidades significativas de calcio como las estudiadas en el apartado 4.4; circunstancias que pueden producirse con facilidad en la industria cerámica, dado que se utilizan materiales orgánicos en la aplicación de esmaltes (vehículos, ligantes, etc.) así como materiales que contienen calcio (aguas duras, fritas, carbonato cálcico, etc.).

\section{CONCLUSIONES}

- Se ha observado que en las condiciones en que se han llevado a cabo los ensayos, la presencia de determinado tipo de materia orgánica y de calcio en soluciones que contienen plomo, aumenta los valores de la $\mathrm{EC}_{50}$ obtenidos mediante el ensayo de inhibición de la luminiscencia de la bacteria Vibrio fischeri. Por tanto, en la caracterización de muestras que contengan materia orgánica y/o calcio, como puede darse en residuos de la industria cerámica, si se utiliza este método de ensayo pueden obtenerse resultados muy diferentes para una misma carga contaminante.

- Al estudiar el efecto de la presencia de diversos elementos típicos de la industria cerámica de productos esmaltados, en concreto de plomo, cinc y boro, sobre el valor de $\mathrm{EC}_{50}$ determinado mediante el ensayo de inhibición, se ha observado que el valor obtenido viene dado fundamentalmente por la concentración del elemento más tóxico para la bacteria, siendo casi independiente de los restantes elementos presentes en la muestra.

- De los factores estudiados, todos contribuyen a disminuir el valor de la toxicidad, por lo que se podría decir que es muy difícil obtener falsos positivos pero sí se pueden obtener falsos negativos. Esto es lo mismo que decir que una muestra caracterizada como tóxica según este ensayo es muy probable que sea verdaderamente tóxica, pero cuando el resultado del ensayo es el contrario puede haber sido interferido por alguno de los factores estudiados.

- Por todo ello, dada la notable influencia del medio a la hora de caracterizar un residuo según el método de inhibición de la luminiscencia, se considera que la caracterización de residuos cerámicos basándose únicamente en los resultados de este ensayo no es suficiente. Para caracterizar la toxicidad de estos residuos el método de caracterización debería complementarse con el análisis químico de la muestra y/o del lixiviado. 


\section{BIBLIOGRAFÍA.}

1. ORDEN DEL 13 DE OCTUBRE DE 1989 por la que se determinan los métodos de caracterización de residuos tóxicos y peligrosos. BOE Nº270 (152/7) del 10 de noviembre de 1989.

2. UNE-EN ISO 11348:1999. Determinación del efecto inhibidor de muestras de agua sobre la luminiscencia de Vibrio fischeri (ensayo de bacterias luminiscentes) Partes 1, 2 y 3.

3. ASTM D 5660-96. Standard Test Method for Assessing the Microbial Detoxification of Chemically Contaminated Water and Soil Using a Toxicity Test with a Lumniscent Marine Bacterium.

4. NF T 90-320:1991. Determination de l'inhibition de la luminiscence de Photobacterium Phosphoreum.

5. DIN 38412:1991. Bestimmung der Hemmwirkung von Abwasser auf die Lichemission von Photobacterium phosphoreum. Leuchtbakterien-Abwasserest mit konservierten Bakterien (L34). Teil 34 .

6. D. Vasseur, F. Bois, J.F. Ferard, C. Rast. «Influence of Physicochemical Parameters on the Microtox Test Response». Toxicity Assessment: An International Quarterly. 1, 283-300 (1986).

7. A.L. Hinwood, M.J. McCornink. «The effect of Ionic Solutes on $\mathrm{EC}_{50}$ Values Measured using the Microtox Test». Toxicity Assessment: An International Quarterly. 2, 449-461 (1987).
8. H.C.H. Hakne, W. Kroontje. «Significance of $\mathrm{pH}$ and Chloride Concentration on Behavior of Heavy Metal Pollutants Mercury (II), Cadmium (II), Zinc (II) and Lead (II)». J. Environ Quality, 2 (4) (1973).

9. E. Monfort, J.E. Enrique, M.F. Gazulla, A. Blasco. «Caracterización de residuos de la industria azulejera». Técn. Cerám., 222, 238-246 (1994).

10. E. Monfort. Desarrollo de un proceso de solidificación-inertización para fangos de la industria cerámica. Estudio cinético de la lixiviación de componentes del producto inertizado. Tesis doctoral. Universitat Jaume I, Castellón 1995.

11. J.E. Enrique, E. Monfort, V. Cantavella, F. Negre. «Leaching kinetics of toxic elements immobilized in cement matrices (I). Selecting a test method». Materials Engineering, 8(2), 151-169 (1997).

12. M.F. Gazulla, M.P. Gómez, A. Barba, E. Monfort, M. Orduña. «A methodology for characterising ceramic wastes». Management of Environmental Quality: An international Journal, 14(3), 333-343 (2003).

13. Santiago Vicente Pérez. «Química de las disoluciones: diagramas y cálculos gráficos». Madrid. Ed. Alhambra, 1979.

14. F. Burriel Martí, et.al. «Química analítica cualitativa».Madrid. Ed. Paraninfo, 1994.

15. IEEE/ASTM SI 10 ${ }^{\mathrm{TM}}-2002$. American National Standard for Use of the International System of Units (SI): The Modern Metric System.

Recibido: 24.09 .03

Aceptado: 16.01 .04 


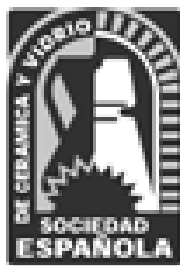

SOCIEDAD ESPAÑOLA DE CERAMICA Y VIDRIO

\section{Boletín de inscripción}

Empresa/Nombre:

Dirección:

Población: Provincia:

C.P. C.I.F./N.I.F:

Tel. Fax:

E-mail:

Persona de contacto:

Productos que fabrica o transforma

Transferencia Bancaria:

Señores les ruego que a partir de ahora y hasta nueva orden cargen a mi cuenta/libreta los recibos que tramite para su cobro la Sociedad Española de Cerámica y Vidrio; Marquen en el cuadro de cuotas el tipo de socio al que pertenece.

El abajo firmante manifiesta que conoce y acepta los términos contenidos en el Estatuto y Reglamento de la Sociedad Española de Cerámica y Vidrio y expresa su deseo explícito de pertenecer a ella en calidad de $\mathrm{SOCIO}$.

Sección de la Sociedad a la que desea pertenecer a efectos de voto:

$\square$ I. Arte y diseño.

$\square$ VI. Materias primas.

$\square$ II. Cerámica blanca, pavimentos y revestimientos cerámicos. $\square$ VII. Refractarios.

$\square$ III. Ciencia básica. $\square$ VIII. Vidrios

$\square$ IV. Esmaltes y pigmentos cerámicos. $\square$ IX. Electrocerámica.

$\square$ V. Ladrillos y tejas. $\quad \square$ X. Medio ambiente

\section{Cuadro de cuotas}

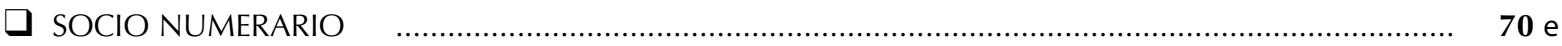

SOCIO CORPORATIVO: $\square$ Pequeñas (hasta 25 empleados) .............................................................. 150 e

$\square$ Medianas (hasta 100 empleados) ........................................................ 300 e

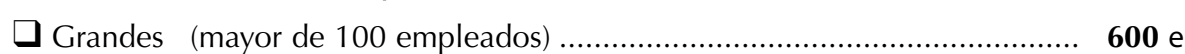

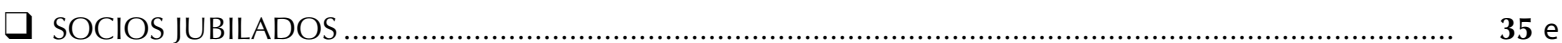

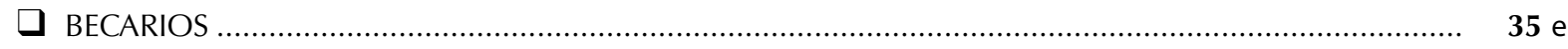

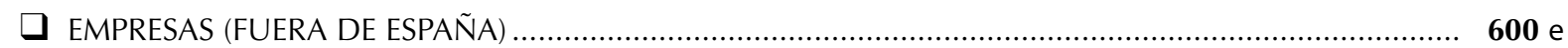

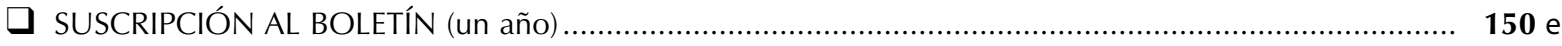

Sociedad Española de Cerámica y Vidrio • Instituto de Cerámica y Vidrio • Camino de Valdelatas s/n • 28049 Madrid

Tfnos.: +34917355840y +34917355860 • Fax: +34917355843

web: www.secv.es • e-mail: secv@icv.csic.es

C.I.F.: G-28200327 